\section{Tranexamic Acid Plus Bemiparin Sodium as Long- Term Prophylaxis in a Patient With FXII-HAE During Pregnancy: A Case Report}

Loli-Ausejo D ${ }^{1}$, Hernández-Martín $\mathrm{I}^{1}$, Cabañas $\mathrm{R}^{1,2,5}$, Entrala $\mathrm{A}^{1,2}$, Gutiérrez-Alvariño $\mathrm{M}^{3}$, Martínez-Sánchez $\mathrm{N}^{4}$, Caballero $\mathrm{T}^{1,2,5}$ ${ }^{1}$ Allergy Department, La Paz University Hospital, Madrid, Spain ${ }^{2}$ Hospital La Paz Institute for Health Research (IdiPaz), Madrid, Spain

${ }^{3}$ Hematology Department, La Paz University Hospital, Madrid, Spain

${ }^{4}$ Obstetrics and Gynecology Department, High Risk Pregnancy Unit, Autoimmune Diseases and Pregnancy clinic, La Paz University Hospital, Madrid, Spain

${ }^{5}$ Biomedical Research Network on Rare Diseases - U754 (CIBERER), Madrid, Spain

J Investig Allergol Clin Immunol 2020; Vol. 30(6): 464-465 doi: $10.18176 /$ jiaci.0585

Key words: Hereditary angioedema. Pregnancy. Treatment. Bemiparin sodium. Tranexamic acid.

Palabras clave: Angioedema hereditario. Embarazo. Tratamiento. Bemiparina sódica. Ácido tranexámico.

Hereditary angioedema (HAE) is characterized by recurrent attacks of severe swelling with involvement of multiple organs. The attacks are induced by genetic mutations that result in increased bradykinin levels. In particular, patients with a mutation in the $F 12$ gene (FXII-HAE) experience worsening of symptoms under hyperestrogenic conditions, such as pregnancy, oral contraceptive intake, and in vitro fertilization [1-3]. Therapy for HAE is limited during pregnancy, delivery, and postpartum $[1,3,4]$, with plasma-derived $\mathrm{C} 1$-inhibitor concentrate (pdC1-INH) being the approach of choice during these periods [3,4].

We report the course and management of repeated angioedema attacks during pregnancy in a 32-year-old woman with FXII-HAE.

The patient had been diagnosed with FXII-HAE at the age of 27, with normal C1-INH levels and function and a single missense mutation in the $F 12$ gene (c.1032C $>$ A). The diagnosis was made in 2013, during her first pregnancy, owing to a severe attack of facial angioedema (previously reported by Gomez-Traseira [5]). She had previously experienced angioedema attacks after starting a combined oral contraceptive pill (Yasmin, drospirenone $3 \mathrm{mg} /$ ethinylestradiol $0.0 \mathrm{mg}$ )

Her second pregnancy was marked by recurrent episodes of facial angioedema starting during the third week of gestation. The patient went on sick leave in the seventh week of gestation and was followed up in the high-risk pregnancy outpatient clinic of our center. During the first trimester, she experienced 3 episodes of facial angioedema (at weeks 3, 5, and 7 of gestation) that required treatment with intravenous pdC1INH
(Berinert, CSL-Behring) in the emergency department. Sometimes she needed 2 or 3 doses of intravenous pdC1INH $1500 \mathrm{IU}$ to resolve the attack.

Given the increased frequency of angioedema attacks, most of which affected the face, and the poor response to high doses of intravenous pdC1INH, we decided to initiate long-term prophylaxis with tranexamic acid (TXA) because of its previously reported usefulness [2,3] and the lack of efficacy of pdC1INH in the treatment of acute attacks in this case. The patient was evaluated by a hematologist to assess hypercoagulable states before initiating treatment with TXA. As pregnancy itself is a physiological prothrombotic state, the assessment was not performed, and the hematologist recommended concomitant treatment with anticoagulants. At 11 weeks of gestation, we initiated long-term prophylaxis with tranexamic acid $500 \mathrm{mg}$ every 8 hours and subcutaneous bemiparin sodium 7500 IU daily. Hematologic monitoring was performed every 5 weeks.

The patient was asymptomatic for 5 months immediately after starting oral long-term prophylaxis with TXA and bemiparin sodium. She experienced only 2 mild facial attacks during the third trimester. One of these was treated with intravenous pdC1INH (1500 IU), and the response was acceptable.

A cesarean delivery was scheduled because of a prior cesarean due to cephalopelvic disproportion [5] at the 39th week of gestation. The patient discontinued TXA the night before the delivery, and short-term prophylaxis with intravenous pdC1INH (1500 IU) was administered before the procedure. The patient also underwent tubal ligation for contraceptive purposes. No complications were reported during pregnancy, cesarean delivery, or postpartum.

The patient gave birth to a healthy girl $(3.5 \mathrm{~kg}$, length $50 \mathrm{~cm}$, and Apgar score of 10 at minutes 1 and 5). An F12 gene mutation was ruled out in the newborn. The patient breastfed her child for 4 months. TXA was not reinitiated, and the patient continued treatment with subcutaneous bemiparin sodium for 6 weeks after delivery. No new HAE attacks were recorded, and the child did not present any drug-related abnormalities. The child is now 18 months old and healthy.

Tranexamic acid was shown to be helpful for preventing FXII-HAE attacks during pregnancy $[2,3,6]$, although there are no reported cases of its association with a heparin as long-term prophylaxis. TXA could be effective for prevention and remission of HAE attacks, because it inhibits activation of FXII owing to its antiplasmin activity and, therefore, the contact activation system [7]. Despite the theoretical increased risk of thrombotic events due to TXA, there are no reported increased risks of thromboembolic events, including myocardial infarction and stroke [1]. Bemiparin sodium is a second-generation low-molecular-weight heparin (LMWH) that is widely used as a thromboprophylactic agent and for clot prevention during hemodialysis. Evidence for the efficacy of heparin to control angioedema attacks in patients with C1-INH-HAE is discordant. A case series of 30 patients with C1-INH-HAE treated with nadroparin, another LMWH, as on-demand therapy and short-term prophylaxis was effective in adults and children, even during pregnancy and postpartum [8]. Enoxaparin, also an LMWH, proved to be safe 
and effective in a pregnant woman with C1-INH-HAE and heterozygous mutations in the MTHFR gene [9]. However, a double-blind placebo-controlled trial involving 24 patients with $\mathrm{C} 1-\mathrm{INH}-\mathrm{HAE}$ failed to attenuate the average intensity of attacks with injected or inhaled calcium heparin as long-term prophylaxis [10].

In the present case, we were able to manage the angioedema attacks during pregnancy using TXA plus bemiparin sodium with no adverse effects. This case demonstrates the importance of individualized treatment during pregnancy in patients with HAE. Consensus on long-term prophylaxis for FXII-HAE is lacking, and clinical trials with FXII-HAE are needed. We expect that future studies will clarify the effectiveness and safety of TXA combined with heparin for patients with FXIIHAE during pregnancy.

\section{Funding}

The authors declare that no funding was received for the present study.

\section{Conflicts of Interest}

Dr D. Loli-Ausejo has received funding to attend conferences/educational events from CSL Behring and Shire (a Takeda company) and is/has been a clinical trial/registry subinvestigator for Shire.

Dr I. Hernández-Martin has received funding to attend conferences/educational events from CSL Behring and Shire and is/has been a clinical trial/registry subinvestigator for Shire.

Dr R. Cabañas has received funding to attend conferences/ educational events from CSL Behring and Shire and is/has been a clinical trial/registry subinvestigator for Biocryst, Novartis, Shire, and CSL-Behring.

Dr A. Entrala has received funding to attend conferences/ educational events from CSL Behring and Shire and is/has been a clinical trial/registry subinvestigator for Biocryst, Novartis, and Shire.

Dr T. Caballero has received research grant support and/ or speaker/consultancy fees from BioCryst, CSL Behring, Merck, Novartis, Octapharma, Pharming, and Shire and funding to attend conferences/educational events from CSL Behring, Novartis, and Shire. She is/has been a clinical trial/ registry investigator for Biocryst, CSL Behring, Novartis, Pharming, and Shire and is a researcher in the IdiPAZ program for promotion of research activities.

Dr M. Gutiérrez-Albariño and Dr N. Martínez-Sánchez declare that they have no conflicts of interest.

\section{References}

1. Caballero T, Farkas H, Bouillet L, Bowen T, Gompel A, Fagerberg $C$, et al. International consensus and practical guidelines on the gynecologic and obstetric management of female patients with hereditary angioedema caused by $\mathrm{C} 1$ inhibitor deficiency. J Allergy Clin Immunol. 2012;129(2):308-20.

2. Marcos C, López Lera $A$, Varela $S$, Liñares $T$, Alvarez-Eire MG, López-Trascasa M. Clinical, biochemical, and genetic characterization of type III hereditary angioedema in 13 Northwest Spanish families. Ann Allergy Asthma Immunol. 2012;109(3):195-200.

3. Caballero T, Canabal J, Rivero-Paparoni D, Cabañas R. Management of Hereditary Angioedema in Pregnant Women: A Review. Int J Womens Health. 2014;6:839-48.

4. García JFB, Takejima P, Veronez CL, Aun MV, Motta AA, Kalil J, et al. Use of pdC1-INH concentrate for long-term prophylaxis during pregnancy in hereditary angioedema with normal C1INH. J Allergy Clin Immunol Pract. 2018;6(4):1406-8.

5. Gómez-Traseira C, Caballero T. Angioedema after caesarean delivery in a patient with hereditary angioedema with normal C1 inhibitor and P.THR309LYS mutation in the F 12 gene is it always bradykinin-mediated? Curr Allergy Clin Immunol. 2017;30(2):94-6.

6. Zuraw BL, Bork K, Binkley KE, Banerji A, Christiansen SC, Castaldo $A$, et al. Hereditary angioedema with normal C1 inhibitor function: consensus of an international expert panel. Allergy Asthma Proc. 2012;33 Suppl 1:S145-56.

7. Feray S, Frain O, Kayemg G, Sabourdin N, Constant I, Rigouzzo A. Repeated attacks of Type III hereditary angioedema with Factor XII mutation during pregnancy. Int J Obstet Anesth. 2018;36:114-8.

8. Majluf-Cruz A, Nieto-Martínez S. Long-term follow up analysis of nadroparin for hereditary angioedema. A preliminary report. Int Immunopharmacol. 2011;11(8):1127-32.

9. Martinez-Saguer I, Escuriola Ettingshausen C. Successful management of hereditary angioedema during pregnancy in a patient with heterozygous MTHFR mutation. Ann Allergy Asthma Immunol. 2017;118(6):734-5.

10. Weiler JM, Quinn SA, Woodworth GG, Brown DD, Layton TA, Maves KK. Does heparin prophylaxis prevent exacerbations of hereditary angioedema? J Allergy Clin Immunol. 2002;109(6):995-1000.

Manuscript received March 13, 2020; accepted for publication May 7, 2020.

David Loli-Ausejo

Allergy Department

La Paz University Hospital

Paseo de la Castellana, 261

28046 Madrid, Spain

E-mail: David.loli.ausejo@gmail.com 